\title{
Developmental Maturation of Calcium Transport by Rat Brush Border Membrane Vesicles
}

\author{
FAYEZ K. GHISHAN AND NOUSHIN ARAB \\ Vanderbilt University, Department of Pediatrics, Division of Gastroenterology, Medical Center North D-4130, \\ Nashville, Tennessee 37232
}

\begin{abstract}
The developmental aspects of calcium transport across the intestinal brush membrane vesicles was studied utilizing a millipore filtration technique. Calcium transport represented uptake into the intravesicular space as evidenced by osmolality studies, calcium release by the calcium ionophore A23187, and temperature dependency. Calcium transport in both suckling and adolescent rats appears to occur by a saturable mechanism. Calcium uptake was similar in the presence of sodium and potassium gradients, but decreased in the presence of choline gradient. The imposition of negative membrane potential did not enhance calcium uptake compared to voltage clamp conditions indicating an electroneutral process. The initial rate of calcium uptake was linear up to $15 \mathrm{~s}$. Kinetic analysis of calcium uptake at $7 \mathrm{~s}$ showed lower $V_{\max }$ and lower $K_{m}$ in suckling rats compared to adolescent rats. These studies are the first to demonstrate the maturational aspects of calcium entry at the brush border level and are consistent with our previous kinetic studies utilizing whole tissue. (Pediatr Res 22: 173-176, 1987)
\end{abstract}

\section{Abbreviation}

BBMV, brush border membrane vesicles

Intestinal calcium transport in the rat has been studied by in vivo perfusion techniques (1), everted gut sacs (2), and Ussing chambers (3). These studies provided the overall picture of calcium absorption and has shown that calcium transport occurs by mediated and nonmediated processes $(1-3)$. The mediated process is regulated by $1-25(\mathrm{OH})_{2}$ vitamin $D_{3}$ the most active polar metabolite of vitamin $D(4)$. The nonmediated process occurs mainly through the paracellular pathway (5). Studies on the developmental aspects of calcium transport using everted gut sacs has shown that the contribution of the nonmediated process to the total process of transport declines with age while the mediated component increases (2). The permeability coefficient of calcium in everted gut sacs was higher in suckling rats compared to adolescent rats. Recently studies using isolated membrane vesicles have provided insight into the process of uptake and exit separately. These membrane vesicles offer the advantage of setting the experimental conditions at will, i.e. defining the composition of the media at both sides of the membrane without interference with cell metabolism (6). We have recently validated the use of brush border membrane vesicles for the study of developmental aspects of transport $(7,8)$.

Using a well-validated technique, we designed the current

Received January 16, 1987; accepted March 2, 1987.

Correspondence and reprint requests Fayez K. Ghishan, M.D., Professor of Pediatrics, Vanderbilt University, Medical Center North, D-4130, Nashville, TN 37232 . studies to examine the developmental aspects of calcium transport across the brush border membrane vesicles of the rat intestine.

\section{METHODS}

${ }^{45} \mathrm{Ca}(14.6-26 \mathrm{mCi} / \mathrm{mg})$ was purchased from New England Nuclear Corp. (Boston, MA). Chemicals were obtained from Sigma Chemical Company (St. Louis, MO). Cellulose nitrate filters, $0.45 \mu \mathrm{M}$ pore size, were obtained from Sartorius Filters, Inc. (Hayward, CA). All chemicals were of the highest purity available.

$B B M V$. Sprague-Dawley rats of varying ages (suckling 14-15 days old and adolescent 42-44 days old) were supplied by Sasco Industries (Omaha, NE) suckling rats were purchased with their mothers. Adolescent rats were shipped directly from the supplier. The animals were housed in the animal care facility. Suckling rats were allowed to suckle freely mother's milk, while adolescent rats were fed standard rat chow (Teklad Diets, Madison, WI) ad libitum until their sacrifice by cervical dislocation. After sacrifice the entire jejunum was removed, washed with ice cold $0.9 \%$ $\mathrm{NaCl}$ and everted over a glass rod. The jejunal region extended from the ligament of Treitz to 20 and $50 \mathrm{~cm}$ aborally for suckling and adolescent rats, respectively. Brush border membrane vesicles were prepared by sequential precipitation with $0.01 \mathrm{M} \mathrm{MgCl}_{2}$ and differential centrifugation as described previously (7-9). The purity of the vesicle preparation for rats of varying ages has been previously demonstrated (7-9).

Transport measurements. Uptake of radiolabeled calcium was measured by a rapid filtration technique. Typically, incubation was initiated by the addition of $20 \mu \mathrm{l}$ of vesicle suspension to 60 $\mu 1$ of incubation solution. Experiments were conducted at $25^{\circ} \mathrm{C}$ unless otherwise stated. After the desired incubation time interval, transport was terminated by abrupt dilution in $1 \mathrm{ml}$ ice cold "stop solution" (100 mM mannitol, $150 \mathrm{mM} \mathrm{MgCl}_{2}$, and $10 \mathrm{mM}$ Hepes/Tris buffer pH 7.4). The reaction mixture was then immediately pipetted onto a prewetted filter (Cellulose Nitrate 0.45 $\mu \mathrm{M}$ pore size, Sartorius Filters, Inc.) and kept under suction. The filter was then rinsed with $5 \mathrm{ml}$ of ice-cold "stop solution" and prepared for scintillation counting. Binding of the radiolabeled calcium to the filter was determined by filtration of incubation media without vesicle protein and was considered as background and substracted from vesicle uptake. Results are expressed as nmol of calcium uptake per mg of vesicle protein. Most experiments were performed in triplicate and repeated at least twice on different days using freshly prepared vesicles. Statistical significance was analyzed by the unpaired Student's $t$ test. $\mathrm{K}_{\mathrm{m}}$ and $\mathrm{V}_{\max }$ were determined according to a computer model for estimation of kinetic parameters (10).

\section{RESULTS}

Calcium uptake versus binding. To determine whether calcium uptake represented binding or transport into the intravesicular 
space, three experiments were conducted. First, the effect of media osmolality was studied in calcium uptake. To determine whether calcium uptake represents transport into osmotically active spaces or mere binding, BBMV from suckling and adolescent rats were incubated in solutions with various osmolarities and calcium uptake was determined under these conditions. Figure 1 shows that at $7 \mathrm{~s}$ calcium uptake into jejunal BBMV from adolescent rats represented uptake into the intravesicular space with small binding component as depicted by the intercept of the relationship of uptake and osmolality $(\mathrm{Y}=0.036+0.17$ $\times, \mathrm{ml}=0.96$ ). Similar relationship was obtained with suckling rat $\mathrm{BBMV}$.

Second, the effect of temperature on calcium uptake was determined by comparing uptake at 25 and $0^{\circ} \mathrm{C}$. As seen in Figure 2, uptake at $0^{\circ} \mathrm{C}$ was significantly decreased compared to values at $25^{\circ} \mathrm{C}$, suggesting that calcium uptake represents an

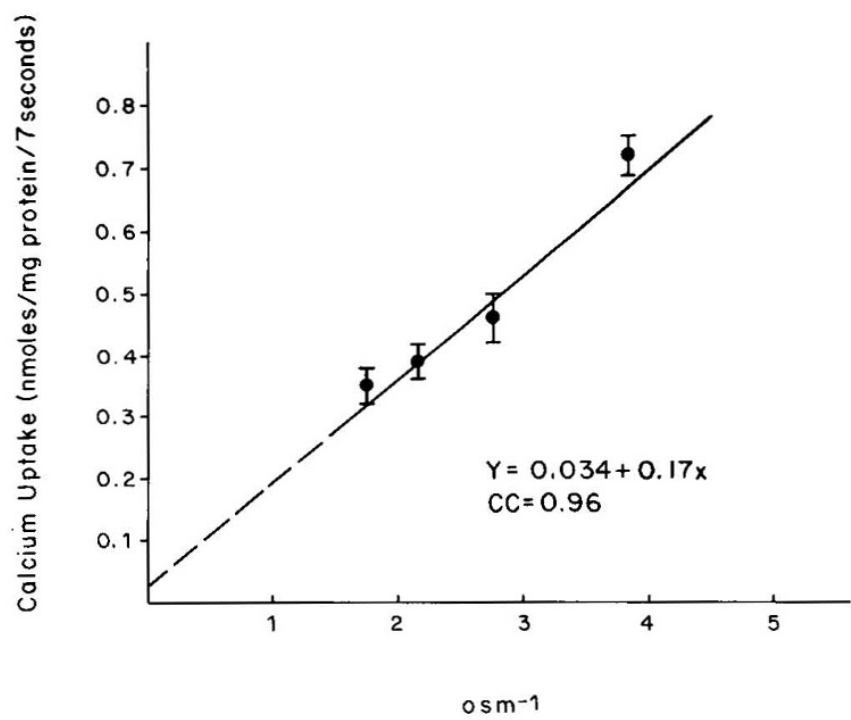

Fig. 1. Effect of media osmolality on calcium uptake. Jejunal BBMV were brought up in $300 \mathrm{mM}$ mannitol and $20 \mathrm{mM}$ Hepes/Tris buffer (pH 7.4). Twenty $\mu \mathrm{l}$ of vesicle preparation was then incubated in incubation media containing $100 \mathrm{mM} \mathrm{NaCl}$ and increasing concentrations of mannitol in an effort to increase the media osmolarity. As seen, there is a linear relationship between media $1 /$ osmol and uptake and at infinite osmolality there is minimal binding, indicating that calcium uptake at 7 $s$ represents transport into the intravesicular space.

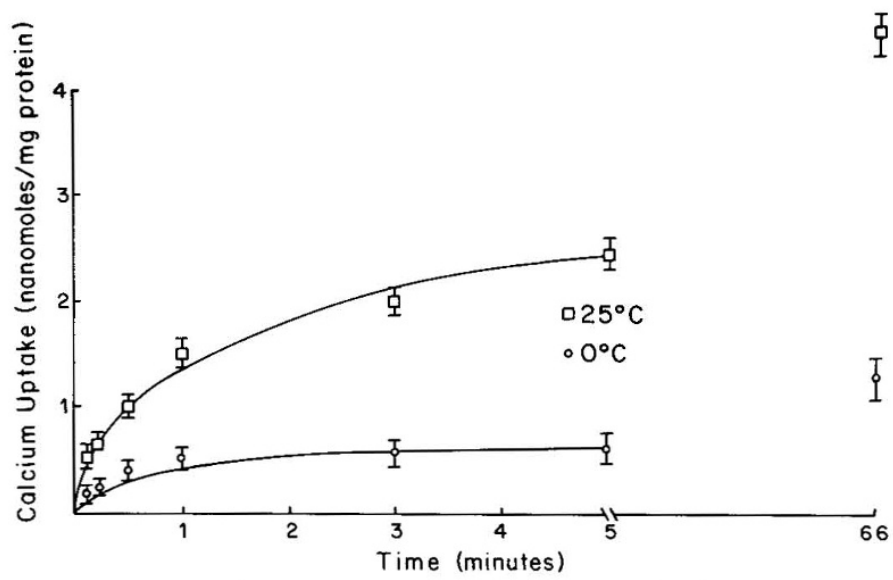

Fig. 2. Effect of temperature on calcium uptake. Calcium uptake was determined in intestinal BBMV from adolescent rats 25 and $0^{\circ} \mathrm{C}$. Values represents mean $\pm \mathrm{SE}$ of $(n=6)$ determinations. Similar results were also obtained in suckling rats.

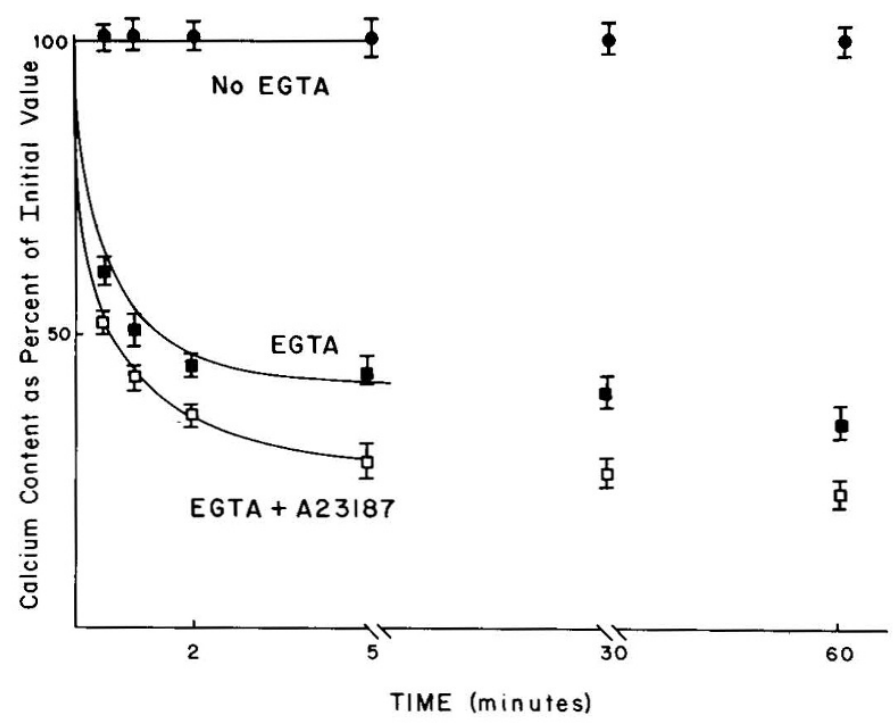

Fig. 3. Effect of calcium ionophore A23187 on calcium efflux. Intestinal BBMV from adolescent rats were loaded into ${ }^{45} \mathrm{Ca}$ for $15 \mathrm{~min}$. Vesicles were then diluted in an incubation media containing calcium ionophore A23187 $10 \mu \mathrm{g} / \mathrm{mg}$ protein with or without $1.5 \mathrm{mM}$ EGTA. Samples were obtained at desired time intervals. Values represents mean \pm SE of $(n=6)$ determinations.

energy dependent process rather than mere binding. Third, calcium ionophore A23187 was added at $10 \mu \mathrm{g} / \mathrm{mg}$ protein to BBMV preincubated for $15 \mathrm{~min}$ in ${ }^{45} \mathrm{Ca}$. As seen in Figure 3, calcium efflux occurred rapidly suggesting that calcium was present in the intravesicular space. These observations were seen in both age groups and suggest that calcium transport represented uptake into the intravesicular space.

Calcium uptake with time. Uptake of calcium was determined as a function of time and cationic gradient. BBMV were prepared in a mannitol buffer and incubated in a sodium, potassium or choline containing buffer so that $100 \mathrm{mM}$ cationic gradient was directed from the extravesicular to the intravesicular space. The osmolalities of the incubation solutions remained similar. Figure 4 depicts calcium uptake with time in the presence of sodium, potassium, and choline gradients. Calcium uptake increased with time and shows saturability in both age groups. There was no significant differences in uptake values in the presence of sodium and potassium gradients, while in the presence of choline gradient uptake was significantly decreased in both age groups. Equilibrium values were similar for both age groups. The capability of suckling and adolescent jejunal vesicle preparation for uphill, sodium-coupled transport has been previously shown (7).

Effect of electrical potential. The effect of an imposed electrochemical membrane potential was studied by valinomycin-induced $\mathrm{K}^{+}$diffusion potential. Membrane potential was induced by preincubating the vesicles with $50 \mathrm{~K}$ gluconate and $200 \mathrm{mM}$ mannitol. The reaction was started by addition of the vesicles to a media containing $10 \mathrm{mM} \mathrm{K}$ gluconate, $280 \mathrm{mM}$ mannitol, and valinomycin. Uptake values were compared to those obtained under voltage clamp conditions, i.e. vesicles preloaded with 50 $\mathrm{mM} \mathrm{K}$ gluconate and $200 \mathrm{mM}$ mannitol ( $\mathrm{K}$ outside $=\mathrm{K}$ inside). Because valinomycin mediates the electrogenic movement of $\mathrm{K}^{+}$ down its concentration gradient, a negative membrane potential is generated. Table 1 shows calcium uptake at $7 \mathrm{~s}, 0.5$, 1 , and 5 min under negative membrane potential and voltage clamp conditions in both age groups. There were no significant differences with negative membrane potential indicating an electroneutral process of uptake.

Initial rate uptake. Figure 5 depicts initial rate uptake of calcium. As seen, calcium uptake was linear up to $15 \mathrm{~s}$. The relationship between calcium uptake and time was $\mathrm{Y}=0.27+$ 

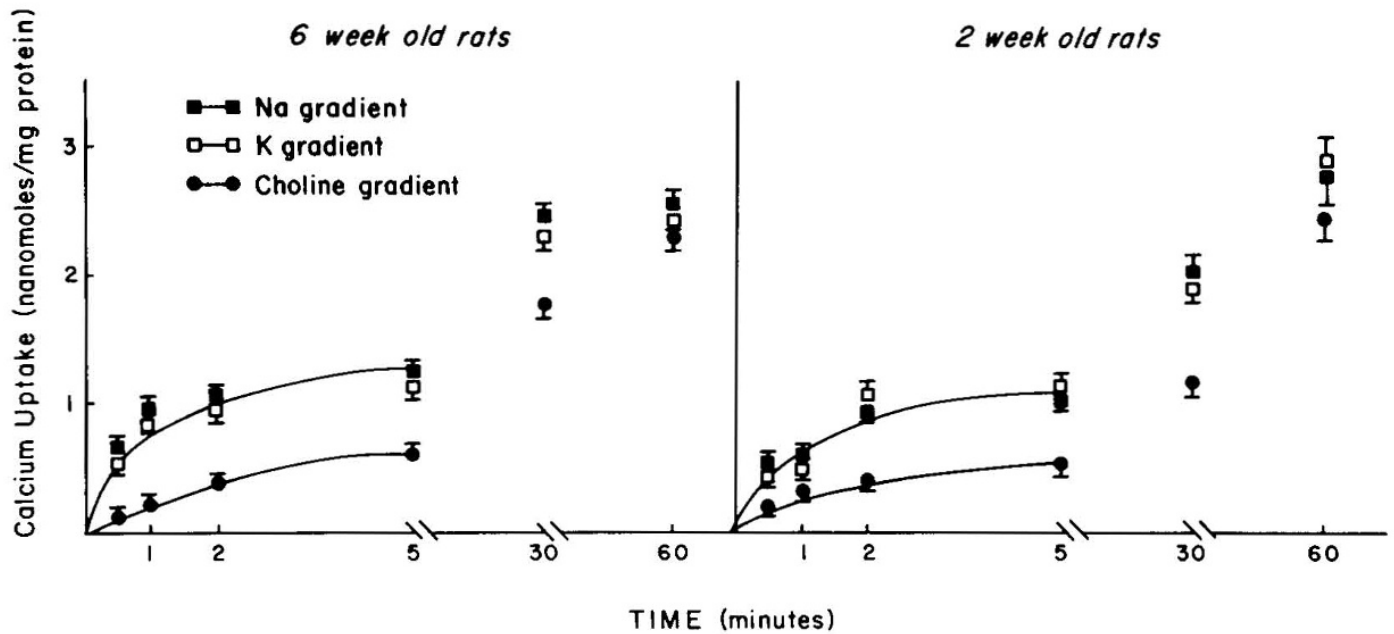

Fig. 4. Effect of cation gradient and time on calcium uptake. BBMV from adolescent and suckling rats were preloaded with $280 \mathrm{mM}$ mannitol and $20 \mathrm{mM}$ Hepes/Tris pH 7.4. Incubations were conducted at $25^{\circ} \mathrm{C}$ in a medium containing either $100 \mathrm{mM} \mathrm{NaCl}$ or $100 \mathrm{mM} \mathrm{KCl}$ or $100 \mathrm{mM}$ choline chloride and $100 \mathrm{mM}$ mannitol, $20 \mathrm{mM}$ Hepes/Tris pH 7.4, and $0.1 \mathrm{mM}^{45} \mathrm{CaCl}_{2}$. Values are mean $\pm \mathrm{SE}$ of $(\mathrm{n}=6)$ determinations.

Table 1. Effect of membrane potential on calcium uptake by BBMV of adolescent rats*

\begin{tabular}{ccc}
\hline Time & Negative membrane potential & Voltage clamp \\
\hline $7 \mathrm{~s}$ & $0.33 \pm 00.1$ & $0.32 \pm 0.001$ \\
$30 \mathrm{~s}$ & $0.92 \pm 0.1$ & $0.97 \pm 0.1$ \\
$1 \mathrm{~min}$ & $1.2 \pm 0.2$ & $1.1 \pm 0.2$ \\
$5 \mathrm{~min}$ & $1.7 \pm 0.3$ & $1.8 \pm 0.2$ \\
\hline
\end{tabular}

* BBMV were preloaded with $50 \mathrm{mM} \mathrm{K}$ gluconate, $200 \mathrm{mM}$ mannitol, and $20 \mathrm{mM}$ Hepes/Tris buffer $\mathrm{pH}$ 7.4. Incubations were started by the addition of vesicles to a media containing either $10 \mathrm{mM} \mathrm{K}$ gluconate, $280 \mathrm{mM}$ mannitol, $20 \mathrm{mM}$ Hepes/Tris buffer $\mathrm{pH} 7.4,0.1 \mathrm{mM}{ }^{45} \mathrm{CaCl}_{2}$, and $10 \mu \mathrm{g}$ protein of valinomycin "voltage clamp" conditions. Values are mean \pm SE of $(n=6)$ determinations. Similar results were obtained with suckling rat brush border membrane preparations.

$0.037 \times$ with correlation coefficient $=0.985$. The positive intercept represents rapid initial binding component (11).

Kinetics of calcium uptake. Kinetics of calcium uptake was determined at $7 \mathrm{~s}$ well within the linear phase of uptake. Figure 6 depicts total uptake of calcium plotted against calcium concentration. $\mathrm{K}_{\mathrm{m}}$ and $\mathrm{V}_{\max }$ for calcium transport in both suckling and adolescent rats was determined. As seen, both $\mathrm{K}_{\mathrm{m}}$ and $\mathrm{V}_{\max }$ were lower in the suckling period compared to corresponding kinetics in adolescent rats $(p<0.01)$.

\section{DISCUSSION}

Calcium transport across the intestinal mucosa occurs via transcellular and paracellular pathways (12). In the classic in vivo perfusion studies and in vitro gut sacs, both pathways are studied together. Moreover, transepithelial calcium transport represents entry at the brush border membrane and transcytosolic movement and exit at the basolateral membrane. Recently, BBMV have been utilized to define events at the entering step of transport without the interference of cellular energy. Our studies utilized a well validated technique to investigate the maturational changes in calcium uptake at the brush border level.

Previous studies in our laboratory have validated the use of BBMV from the jejunum and ileum of rats during maturation (7-9). These studies included morphological, biochemical, and functional criteria which validated the use of these vesicles for transport studies during maturation (7-9).

In order to define whether calcium uptake represent binding or transport into the vesicular space, three studies were con-

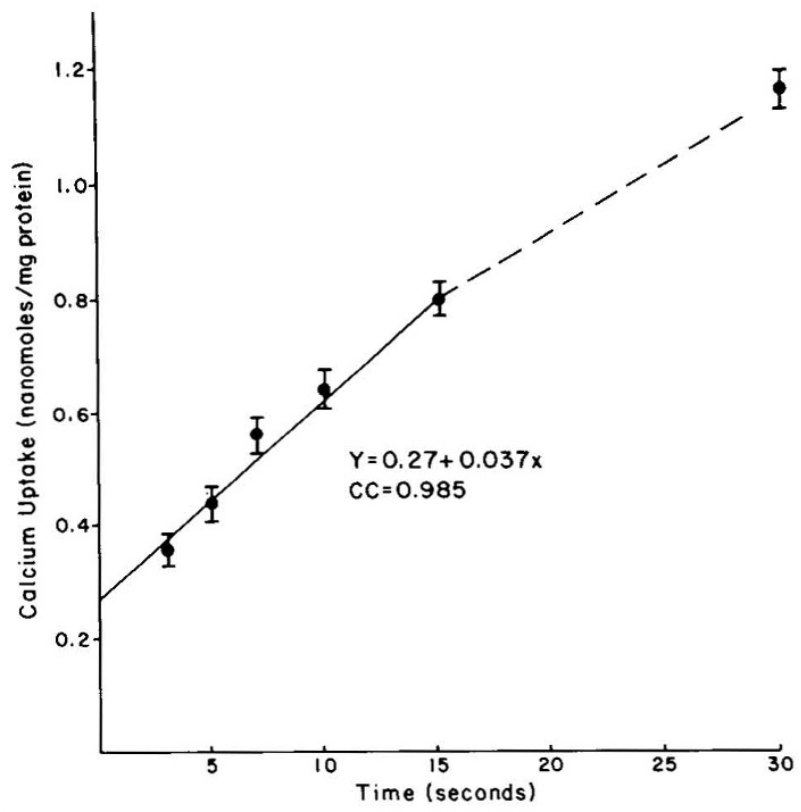

Fig. 5. Initial rate of uptake of calcium. BBMV were brought up in mannitol buffer $\mathrm{pH}$ 7.4. Calcium concentrătion was $0.1 \mathrm{mM}$. Calcium uptake was determined up to $30 \mathrm{~s}$. The uptake appears linear up to $15 \mathrm{~s}$. Values are mean \pm SE of $(n=6)$ determinations.

ducted. First an osmolality experiment was conducted. In this setting, calcium uptake was determined at $7 \mathrm{~s}$ in a media with increasing osmolality. Calcium binding at this early time point represented mostly transport into the intravesicular space with a small binding component. The relationship between calcium uptake and $1 /$ osmol is expressed as $\mathrm{Y}=0.036+0.17 \mathrm{X}$ with correlation coefficient $=0.96$. Thus at isotonicity only $15 \%$ of uptake represented binding. In the second experiment calcium uptake was determined at 25 and $0^{\circ} \mathrm{C}$. The decrease in calcium transport at $0^{\circ} \mathrm{C}$ compared to $25^{\circ} \mathrm{C}$ is consistent with the concept that a major component of calcium uptake represents transport into the intravesicular space rather than binding. Carrier-mediated transport phenomena in general are temperature dependent (13). In the third experiment, calcium ionophore A23187 was added to vesicles preloaded with ${ }^{45} \mathrm{Ca}$. The presence of A23187 enhanced calcium release compared to EGTA indicating the presence of calcium in the intravesicular space. These findings 


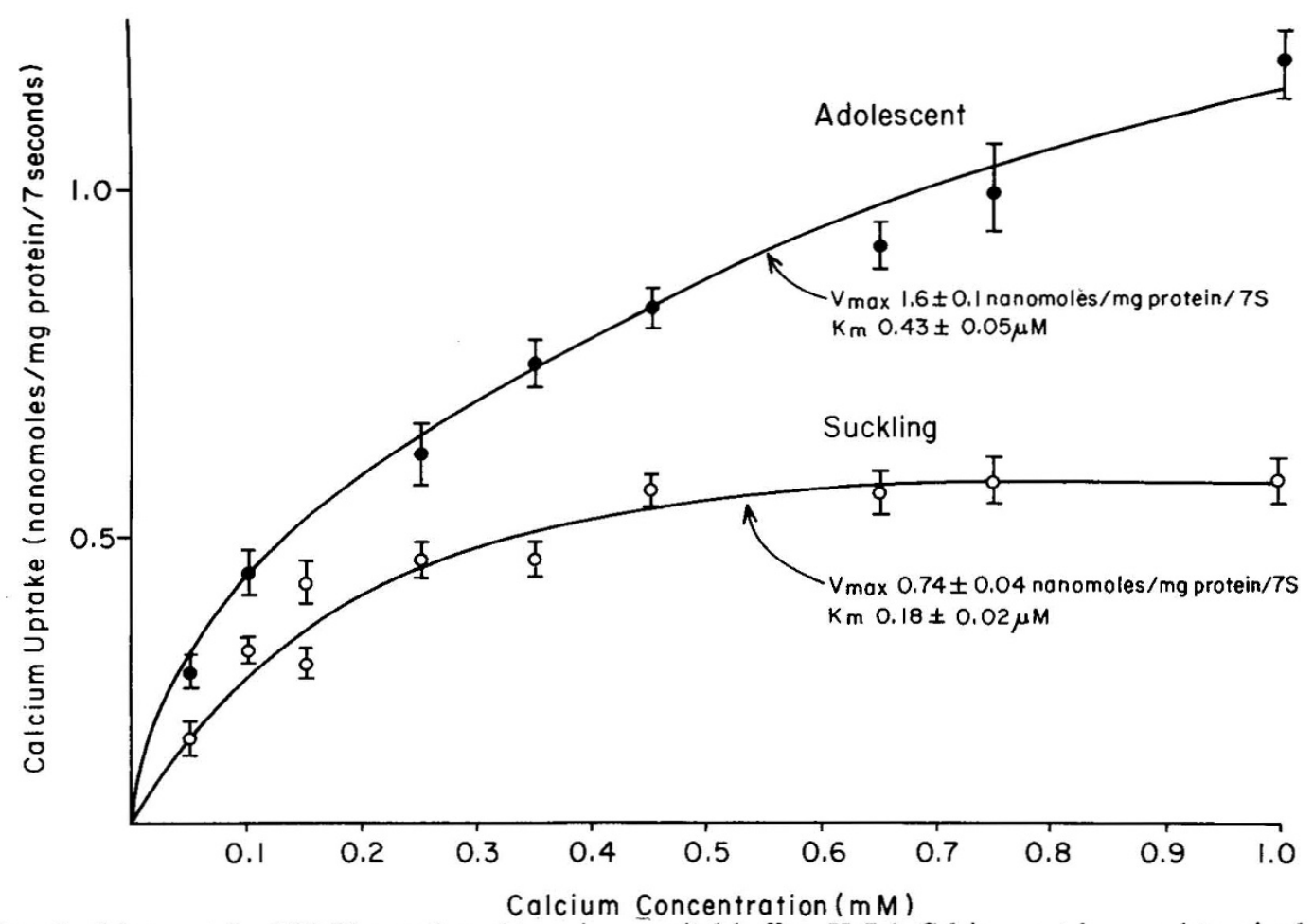

Fig. 6. Kinetics of calcium uptake. BBMV were brought up in mannitol buffer pH 7.4. Calcium uptake was determined at $7 \mathrm{~s}$. Calcium concentration in the media ranged from $0.1-1 \mathrm{mM}$. Kinetic parameters were obtained using a computerized model of the Michaelis-Menten kinetics.

are similar to those of Schedl and Wilson (14). To determine the driving force for calcium uptake by BBMV, uptake studies were done in the presence and absence of sodium gradient. As seen in Figure 4 calcium uptake was similar with $\mathrm{Na}^{+}$or $\mathrm{K}^{+}$gradient indicating that sodium gradient is not the driving force for calcium uptake. Choline gradient depressed calcium uptake as compared to $\mathrm{Na}^{+}$or $\mathrm{K}^{+}$gradient conditions. Calcium uptake, however, showed saturability with time indicating a facilitated diffusion or a carrier-mediated process. These findings are similar to that of Miller and Bronner (15) in which calcium uptake was not sodium dependent and decreased in the presence of choline. An imposed electrical potential as induced by the ionophore valinomycin had no effect on calcium uptake indicating an electroneutral process. A similar process was found with phosphate uptake across the brush border membranes (9). In contrast, D-glucose uptake in this setting was significantly enhanced compared to $\mathrm{Na}^{+}$gradient conditions alone suggesting an electrogenic process (7). Miller and Bronner (15) found slight increase in calcium uptake at $15 \mathrm{~min}$ under negative membrane potential but no increase at $0.5 \mathrm{~min}$. The kinetics of calcium uptake was determined after the demonstration of the linearity of uptake up to $15 \mathrm{~s}$. Therefore kinetics of calcium uptake were determined at $7 \mathrm{~s}$ well within the linearity of uptake. $\mathrm{K}_{\mathrm{m}}$ and $\mathrm{V}_{\max }$ were lower in suckling rats compared with those of adolescent rats, indicating greater affinity but lower capacity in the infant rats compared to adolescent rats. These kinetic parameters are similar to our whole tissue studies in which the $\mathrm{V}_{\max }$ and $\mathrm{K}_{\mathrm{m}}$ of the mediated (i.e. transcellular component) was small in suckling rats and increased with age. Thus, events at the brush border membrane reflects observations with whole tissue.

Our previous in vivo (1) and in vitro gut sac (2) studies indicated that the intestinal calcium transport in the suckling period occurs mainly by a passive process through the paracellular pathway. The transcellular movement was small and increased with age. This study using BBMV representing transcellular movement, agrees with our previous studies, indicating an increase in transcellular calcium transport with advancing age.
In summary, our data suggest that calcium uptake into brush border membranes represents uptake into the intravesicular space. This process is electroneutral, saturable, and not sodium dependent. The kinetic parameters of this process show maturational changes which evolve with age, and are similar to events seen with whole tissue.

\section{REFERENCES}

1. Ghishan FK, Jenkins JT, Younoszai MK 1980 Maturation of calcium transport in the rat small and large intestine. J Nutr 110:1622-1628

2. Ghishan FK, Parker PH, Nichols S, Hoyumpa A 1984 Kinetics of intestinal calcium transport during maturation in the rat. Pediatr Res 18:235-239

3. Walling MW, Rothman SS 1969 Phosphate independent carrier mediated active transport of calcium by rat intestine. Am J Physiol 217:1144-1149

4. Ribovich ML, Deluca HF 1975 The influence of dietary calcium and phosphorus on intestinal calcium transport in rats given vitamin D metabolites. Arch Biochem Biophys 170:1039-1042

5. Nellans HN, Kimberg DV 1978 Cellular and paracellular calcium transport in rat ileum: effects of dietary calcium. Am J Physiol 235:E726-E737

6. Hopfer U 1977 Isolated membrane vesicles as tools for analysis of epithelial transport. Am J Physiol 233:E445-E449

7. Ghishan FK, Wilson FA 1985 Developmental maturation of D-glucose transport by rat jejunal brush border membrane vesicles. Am J Physiol 248:G87G92

8. Barnard JA, Ghishan FK, Wilson FA 1985 Ontogenesis of taurocholate transport by rat ileal brush border membrane vesicles. J Clin Invest 75:869-873

9. Borowitz SM, Ghishan FK 1985 Maturation of jejunal phosphate transport by rat brush border membrane vesicles. Pediatric Res 19:1308-1313

10. Vaughn WK, Neal RA, Anderson AJ 1976 Computer estimation of the parameter of the sigmoid kinetic model. Comput Biol Med 6:1-5

11. Gunther RD, Wright EM $1983 \mathrm{Na}^{+}, \mathrm{Li}^{+}$and $\mathrm{Cl}^{-}$transport by brush border membranes from rabbit jejunum. J Membr Biol 74:85-94

12. Nellans HN, Kimberg DV 1979 Intestinal calcium transport absorption, secretion and vitamin D. In: Crane RK (ed) International Review of Physiology. Gastrointestinal Physiology III. University Park Press, Baltimore, pp 227261

13. DeSmedt H, Kinne R 1981 Temperature dependence of solute transport and enzyme activities in hog renal brush border membrane vesicles. Biochim Biophys Acta 648:247-253

14. Schedl HP, Wilson HD 1985 Calcium uptake by intestinal brush border membrane vesicles. J Clin Invest 76:1871-1878

15. Miller A III, Bronner F 1981 Calcium uptake by isolated brush border membranes in the rat. Biochem J 196:391-401 